\title{
BMJ Open Longitudinal qualitative exploration of cancer information-seeking experiences across the disease trajectory: the INFO-SEEK protocol
}

\author{
Evi Germeni, ${ }^{1}$ Monica Bianchi, ${ }^{2}$ Dario Valcarenghi, ${ }^{3}$ Peter J Schulz ${ }^{1}$
}

To cite: Germeni E, Bianchi M, Valcarenghi D, et al. Longitudinal qualitative exploration of cancer information-seeking experiences across the disease trajectory: the INFO-SEEK protocol. BMJ Open 2015;5:e008933. doi:10.1136/bmjopen-2015008933

- Prepublication history for this paper is available online. To view these files please visit the journal online (http://dx.doi.org/10.1136/ bmjopen-2015-008933).

Received 29 May 2015 Revised 3 September 2015 Accepted 11 September 2015

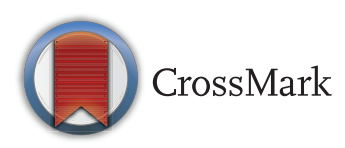

${ }^{1}$ Institute of Communication and Health, Faculty of Communication Sciences, University of Lugano, Lugano, Switzerland ${ }^{2}$ Department of Business Economics, Health and Social Care, University of Applied Sciences and Arts of Southern Switzerland (SUPSI), Manno, Switzerland ${ }^{3}$ Oncology Institute of Southern Switzerland (IOSI), Bellinzona, Switzerland

Correspondence to Dr Evi Germeni; evdokia.germeni@usi.ch

\section{ABSTRACT}

Introduction: A substantial corpus of literature has sought to describe the information-seeking behaviour of patients with cancer. Yet, available evidence comes mainly from cross-sectional studies, which provide 'snapshots' of patients' information needs and information-seeking styles at a single time point. Only a few longitudinal studies currently exist; however, these are quantitative in nature and, despite successfully documenting changes in patients' information needs throughout the clinical course of cancer, they have failed to provide an evidence-based interpretation of the causes and consequences of change. The goal of this study is threefold: First, we wish to provide a holistic understanding of how cancer information-seeking behaviour may evolve across different stages of the patient journey. Second, we will seek to elucidate the contextual and intervening conditions that may affect possible changes in information seeking. Third, we will attempt to identify what the consequences of these changes are, while heightening their implications for clinical practice and policy.

Methods and analysis: We will carry out a longitudinal qualitative study, based on face-to-face, in-depth interviews with approximately 25 individuals diagnosed with cancer. Patients will be recruited from 2 oncology hospitals located in Ticino, Switzerland, and will be interviewed at 3 different time points: (1) within 2 weeks after receiving the cancer diagnosis; (2) within 2 weeks after their initial treatment; and (3) 6 months after their initial treatment. All interviews will be recorded and transcribed verbatim. A grounded theory approach will be used for the analysis of the data. Ethics and dissemination: The study protocol has been approved by the Ethics Committee of Canton Ticino (CE 2813). Participation in the study will be voluntary, and confidentiality and anonymity ensured. Prior to study participation, patients will be asked to provide signed informed consent. Findings will be disseminated in international peer-reviewed journals and presented in relevant conferences.

\section{INTRODUCTION}

Over the past 25 years, health informationseeking behaviour has emerged as an
Strengths and limitations of this study

- There is a striking lack of longitudinal studies examining information-seeking behaviour of patients with cancer.

- Longitudinal qualitative research is an innovative methodological approach that can capture processes involved in change.

- Longitudinal designs are typically prone to high dropout rates of participants.

important concept in the field of health communication. Within the context of a cancer diagnosis, cancer information-seeking behaviour refers to the purposive or goaldriven acquisition of cancer-related information and has been identified as a key coping strategy that can enable individuals to deal with stressful illness-related events, such as the shock of initial diagnosis. ${ }^{1}$ Typically viewed as a problem-focused coping strategy, which can help individuals understand what is at stake and determine what kind of resources and options are available to them for managing the stressors, cancer information seeking has also been shown to have emotion-focused coping functions, as it can reduce negative emotions linked to the uncertainty of the disease and provide reassurance. ${ }^{2}$ Recently, the concept has also gained ground, since it is consistent with the paradigm shift towards patient empowerment and shared decision-making: patients seeking out information have the potential to better evaluate the risks and benefits of different treatment options and can take an active role in medical decision-making. ${ }^{3}$

Research on cancer information seeking has traditionally focused on the type and amount of information that patients want to have, the information sources that they are using, the motivations for seeking information, as well as the outcomes or 
consequences of the search. Although physicians seem to be the most frequent and trusted source of information, it is now well established that the majority of patients with cancer turn to a variety of sources to satisfy their queries. ${ }^{5-7}$ These typically include: print or broadcast traditional media (eg, newspapers, books, brochures, television, radio), new media (eg, internet), and non-medical interpersonal sources (eg, family, friends, other patients)..$^{5-10}$ According to Nagler et al, ${ }^{6}$ there are five main reasons that can explain patients' desire for cross-source engagement: (1) verification, that is, patients' double-checking information from one source by going to a second source; (2) clarification/elaboration, which has to do with patients' need for additional or more detailed information; (3) emotional support, focusing on patients' desire to fulfil support-related needs; (4) directed contact, referring to instances in which one source explicitly directs patients to another source; and (5) proxy/surrogacy, referring to instances in which non-medical interpersonal sources seek information on behalf of the patient, thereby serving as an information proxy or surrogate. Furthermore, despite frequently cited disease-specific and treatment-specific information needs, type of information sought seems to vary depending on the stage of the patient pathway. For instance, issues related to recovery may become prominent only during the post-treatment period. ${ }^{7}$

Not all individuals, however, facing a life-changing diagnosis of cancer decide to search for information beyond what is offered by the treating healthcare professionals. Several studies have documented that a considerable proportion of patients-estimated to range, in developed countries, from $10 \%$ to $30 \%$-avoids further information for fear of mental discomfort or dissonance, whereas there is abundant theoretical and empirical literature on the characteristics that differentiate an information seeker from an information avoider. ${ }^{1}{ }^{11-14}$ In general, both personal and contextual factors seem to influence individuals' willingness to actively search for cancer-related information. The former typically refer to sociodemographic characteristics (eg, age, gender, socioeconomic status, education level $)^{13}{ }^{15-18}$ and psychosocial variables (eg, perceived normative pressure, high internal locus of control, self-efficacy), ${ }^{19-21}$ while the latter may include, but are certainly not limited to, disease-related characteristics (eg, type and stage of cancer, time since diagnosis) ${ }^{5} 181922$ and the context of cancer care. ${ }^{2324}$

Inarguably, the dichotomy of information seeker versus non-seeker still prevails not only in published empirical studies, but also in existing theoretical literature. The most frequently cited theories and models of health information-seeking behaviour (eg, Miller's ${ }^{14}$ monitoring and blunting hypothesis, Lazarus and Folkman's ${ }^{25}$ stress, appraisal and coping theory, Johnson's ${ }^{26}$ comprehensive model of information seeking, Lenz's ${ }^{27}$ information-seeking model) seem to focus on whether individuals decide to search for information or not. Yet, it is growingly recognised that cancer information seeking is a much more complex and multidimensional behaviour than previously considered. Analysing data from a cross-sectional qualitative study, Lambert et al, ${ }^{23}{ }^{24}$ for instance, identified five different patterns of cancer information-seeking behaviour: (1) intense information seeking-a keen interest in detailed cancer information; (2) complementary information seeking - the process of getting 'good enough' cancer information; (3) fortuitous information seeking -the search for cancer information mainly from others diagnosed with cancer; (4) minimal information seeking - a limited interest for cancer information and (5) guided information seeking - the avoidance of some cancer information. At the same time, evidence coming from longitudinal quantitative studies suggests that cancer information-seeking behaviour does not remain stable over time. Eheman $e t a l,^{28}$ for example, found that patients are more active in seeking information before being treated than afterward, whereas Vogel $e t a l^{29}$ showed that patients have the highest information needs at the beginning of treatment, with an evident decrease of needs in the course of treatment. Similarly, synthesising data from various published qualitative studies, Germeni and Schulz ${ }^{30}$ concluded that the boundaries between seeking and avoiding information throughout the journey of patient with cancer are often fluid; therefore, information seeking and avoidance should not be necessarily viewed as two distinct behaviours pertaining to different groups of patients.

Understanding what patients want to know about their cancer and when they need to know it in the course of the disease is fundamental for developing tailored information interventions and ensuring the delivery of quality cancer care. Nonetheless, it could also be a key parameter for the optimisation of the outcomes of cancer information seeking, as despite the frequently reported benefits of searching for cancer-related information (eg, increased involvement in medical decisionmaking, greater satisfaction with treatment, improved coping skills), there is also evidence to suggest that inconsistencies between desired and obtained information may lead to negative outcomes, such as increased levels of anxiety. ${ }^{31}{ }^{32}$ Therefore, it is crucial that relevant research takes into consideration the individual experiences of patients, while providing insightful and contextspecific evidence that could guide healthcare providers' assessments of differential and changing information needs of patients with cancer.

\section{Gaps in existing literature}

As evident from the above, a substantial corpus of literature has sought to describe the information-seeking behaviour of patients withcancer. Yet, available evidence comes mainly from cross-sectional studies, which provide 'snapshots' of patients' information needs and information-seeking styles at a single point in time. Although several published reviews of relevant literature 
have stressed the need for longitudinal research on the topic, ${ }^{70}$ there is currently a striking lack of longitudinal investigations concerning information-seeking behaviour of patients with cancer. What is more, existing longitudinal studies are quantitative in nature and, despite successfully documenting changes in patients' information needs throughout the clinical course of cancer, they have failed to provide an evidence-based interpretation of the causes and consequences of change. Thus, the goal of this longitudinal qualitative study is threefold: First, we wish to provide a holistic understanding of how cancer information-seeking behaviour may evolve across different stages of the patient journey. Second, we will seek to elucidate the contextual and intervening conditions that may affect possible changes in cancer information seeking. Third, we will attempt to identify what the consequences of these changes are, while heightening their implications for clinical practice and policy.

\section{METHODS AND ANALYSIS}

\section{Study design and setting}

We will carry out a prospective, longitudinal qualitative study, based on face-to-face, in-depth interviews with approximately 25 newly diagnosed patients with cancer residing in the Italian-speaking part of Switzerland, namely Ticino. Qualitative research can offer valuable insights into patient experiences, whereas longitudinal qualitative designs have the considerable advantage of addressing questions about how and why these experiences change over time. In-depth interviews were chosen as the most appropriate data collection method, as they can permit exploration of participant experiences in greater depth than allowed by other methods (eg, focus groups). Written informed consent will be obtained from all interviewees prior to study participation.

\section{Recruitment and sampling}

Study participants will be recruited from two oncology hospitals located in the two largest cities of Ticino, that is, Lugano and Bellinzona. We will liaise with clinical staff to identify individuals meeting the predefined eligibility criteria shown in box 1 . We will contact by phone patients showing an initial interest in the study (as this described by the clinical staff) and giving permission to be contacted, to provide further details about the process and, when possible, set up a convenient date and time for the interview. In the initial stages of the study, we will recruit all individuals meeting our inclusion criteria. As the study unfolds, we will employ a theoretical (or analysis-driven purposeful) sampling strategy, ${ }^{33}$ which will involve recruiting additional participants with potentially different cancer informationseeking experiences from those already recruited. We estimate that a sample size of 25 participants will be adequate to reach data saturation; yet, we plan to
Box 1 Eligibility criteria for participation in the INFO-SEEK study

Inclusion criteria

1. Patients aged $\geq 18$ years

2. Physically and cognitively able to participate in the interview process

3. First time cancer diagnosis

4. No evidence of metastases

Exclusion criteria

1. Unable to fulfil the inclusion criteria

2. Unable to provide written informed consent

3. Non-Italian speaking

continue the interviews until the data set is complete, as indicated by data replication or redundancy. ${ }^{34}$

\section{Data collection}

Data collection is estimated to start in September 2015. As issues of time and timing are of paramount importance in longitudinal qualitative research, we have opted for identifying time points by key transitions in the patient's journey rather than having the same time points for all participants. Therefore, two members of the research team (MB and DV) will interview patients at three different time points: (1) within 2 weeks after receiving the cancer diagnosis; (2) within 2 weeks after their initial treatment (eg, surgery, chemotherapy) and (3) 6 months after the initial treatment. Prior to each interview, researchers will clarify that the interviews do not constitute part of the cancer management process and that possible withdrawal will in no way affect the care or treatment patients receive in the hospital. Initial interviews with each patient will begin as follows: "This study is about further information that newly diagnosed patients may wish to have or not about their cancer. Could you tell me what you think about searching for cancer-related information from sources other than your treating doctor and describe your own cancer information-seeking experiences, if any?" Subsequent interviews will begin by asking the participants to reflect on what has changed (in terms of desire and search for cancer-related information) since the last interview. In all cases, further questions from the interviewers will be based on what participants say and will consist mostly of clarification and probing for details. ${ }^{35}$ To ensure that all relevant topics will be covered, we have developed a basic interview topic guide, which includes the following: motivations for seeking (or not seeking) cancer-related information, preferred sources of information, type and amount of information sought, and perceived outcomes of the search. The guide was formulated on the basis of both available literature and our own experience and interest in the topic. Interviews will be conducted at the hospital, in a one-to-one setting, with only the researcher and the patient being present. The estimated duration of the interviews is about $45 \mathrm{~min}$, but this is 
likely to vary depending on the interviewee and the order of the interviews. We expect, for instance, that the first interviews with each patient may be shorter than the subsequent ones, given that participants will have just received a life-changing diagnosis of cancer. Apart from this, involvement over time is likely to enhance trust and facilitate disclosure of information.

\section{Data analysis}

Consistent with a grounded theory approach, ${ }^{36}$ data collection and analysis will proceed concurrently. With participant permission, interviews will be recorded and transcribed verbatim. All transcripts will be checked back against the original audio recordings to ensure content accuracy. Each interview will be accompanied by a brief summary that will outline the main points of the discussion. Similarly, on completion of data collection, case-based summaries will be written as a way of capturing change over time. Data analysis will rely on a threestage coding process that will consist of: (1) initial coding, that is, an intense, line-by-line analysis of the transcripts; (2) focused coding, in which a selected set of most prevalent codes will be identified; and (3) theoretical coding, in which we will refine the final categories in our theory and relate them to one another. ${ }^{37}$ Throughout the study, memo-writing will be used to stimulate and record our developing thinking about the data. Memos will include both operational notes about the data collection process and conceptual memos about the initial codes and focused codes being developed. The final outcome of our analysis will be a theoretical framework, which will account adequately for all gathered data, while seeking to explain the process of cancer information seeking across the disease trajectory.

\section{ETHICS AND DISSEMINATION \\ Ethical considerations}

Ethical issues can be particularly relevant in longitudinal research, especially in cases where the study population involves patients facing a life-threatening disease. Previous studies have identified a number of risks, which can arise from longitudinal qualitative data collection, including intrusion into people's lives, dependency, as well as distortion of experience due to repeated contact, personal involvement and closure of relationships. ${ }^{38-40}$ Therefore, prior to the start of study, we have had several brainstorming sessions to reflect on possible challenges that we may encounter (before, during or after the data collection process) and come up with concrete ways of addressing them. These focus primarily on the following:

\section{Recruitment at critical stages of the patient pathway}

We wish to interview individuals soon after receiving a cancer diagnosis and we intend to repeat the same process shortly after their initial treatment, and then, after 6 months. We recognise that recruitment at these critical stages of the patient pathway can be difficult; yet, there is evidence to suggest that patients often feel more comfortable voicing their internal fears and distress to a researcher rather than a clinician or even people from their close environment. ${ }^{40}$ In this sense, we anticipate that contributing to the research process and talking about the experience of such important life events (at the moment they occur) could even have a cathartic role for some of the patients. Despite this, we have sought to establish a number of strategies, to ensure that study participants will be adequately safeguarded. First, participation in the study will be voluntary and participants will be free to withdraw at any time without giving reasons and without any penalty. Second, consent will be an ongoing process. Specifically, prior to the first interview, participants will be informed about the study objectives, ground rules (limited access to the data, anonymity, confidentiality, etc) and timeline, and will be asked to provide written informed consent. Apart from this, oral consent will also be sought prior to each subsequent interview, as well as during the interviews, where appropriate (eg, if a participant becomes frustrated or emotionally overwhelmed). Last, all interviews will be conducted by two experienced cancer nurses (MB and DV) who are currently conducting their $\mathrm{PhD}$ studies. This is expected to facilitate sensitive recruitment of participants and nurture an atmosphere of trust and reciprocal rapport that is essential for establishing a long-term commitment to the study.

\section{Researcher burnout}

Collecting in-depth data from the same individuals over a prolonged period of time may not affect just the participants; it can also place considerable demands on the researchers. Despite sufficient training and experience, conducting qualitative research with vulnerable population groups can be emotionally challenging. ${ }^{38}$ Participants share their personal stories, disclose intimate thoughts and feelings, and could even reveal to the researchers information that they have not discussed with anyone else. Involvement over time creates a bond between the participant and the interviewer, and although the impact of this on the safety and well-being of the researched has been widely discussed, ethical aspects related to the emotional well-being of the researcher are often overlooked. Talking about 'researcher saturation', Wray et at $t^{41}$ showed how researchers who were involved in all phases of emotionally demanding qualitative research, namely data collection, transcription and analysis, repeatedly relived the stressful events that participants had narrated and proposed a more formal approach for dealing with researcher distress, such as professional counselling. As we cannot afford such possibility, we opted for splitting the research tasks within our group in a 'balanced' way (ie, MB and DV will collect the data, two research assistants will transcribe the interviews, and EG and PJS will perform the data analysis), so that we minimise the level 
of involvement for each single researcher. Regular meetings, promoting debriefing and informal peer support, have also been envisaged to ensure that all members of the research group will be well supported in their role.

\section{Leaving the field}

Although collaborative relationships between the researcher and the researched are beneficial (perhaps, even necessary), the difficulties created by such relationships in the leaving process are seldom considered. Ortiz $^{42}$ has argued that the same skills and techniques that are essential in gathering rich data, like, for instance, rapport building or use of self-disclosure, can also make the exiting process rather stressful. It is, therefore, an issue of research ethics how the field relations will be terminated. Indeed, based on previous experience with family caregivers of patients diagnosed with cancer, ${ }^{43}$ we expect that the closure of relationships will be difficult, not only for the participants, but also for the interviewers. To make this process as smooth as possible, individuals choosing to participate in the study will be provided from the beginning with clear and concrete information about how many times and when they will be approached for an interview. Furthermore, at the end of the last interview, interviewers will thank participants for their contribution to the study and give them a symbolic gift as a small token of appreciation. In the unfortunate event of a patient's death, while the study is ongoing, a condolence card will be sent to the bereaved family. Likewise, to minimise the potential harm that researchers may be exposed to as part of the fieldwork, at the end of the data collection process, interviewers will be provided with opportunities for debriefing as a way of sharing their research stories with the rest of the team, expressing their feelings and concerns, and reflecting on possible ways of managing emotional attachment.

\section{Dissemination of findings}

We will use a variety of methods to ensure that our work will achieve maximum visibility, not only to academic, but also to clinical and policy audiences. Publication of our study protocol provides an important first step towards this direction. In this paper, we have sought to offer a comprehensive overview of relevant literature, while underlining current research gaps that necessitated the design and implementation of the INFO-SEEK study. We have also provided a detailed description of the methodology that we will employ, as well as concrete ways of addressing both participant-related and researcher-related ethical issues, which are likely to arise when conducting longitudinal qualitative research on sensitive or difficult topics.

In the same way, study findings will be disseminated in high-quality, peer-reviewed journals and presented in key national and international conferences. As interest in the research to practice gap has substantially increased, a key element in our dissemination endeavours will be to successfully transfer our research findings to healthcare practice and policy-making. On completion of the project, we intend to organise a local dissemination event and bring together relevant stakeholders (eg, service users, healthcare professionals, hospital managers) to jointly explore ways in which project results can be better integrated into routine clinical practice. Participant experiences of cancer information seeking, for instance, could inform the development of a screening tool for assessing patients' information needs and preferences prior to the medical consultation. The benefits of properly evaluating the amount and type of information that a patient desires throughout different phases of care could be manifold: more effective and targeted information provision (without increasing consultation duration), guidance to using evidence-based secondary information sources, as well as provision of appropriate treatment decision aids.

Contributors EG conceived the study and wrote the initial draft. MB, DV and PJS provided comments and feedback. All authors have read and approved the final version of the manuscript.

Competing interests None declared.

Ethics approval Ethics Committee of Canton Ticino.

Provenance and peer review Not commissioned; externally peer reviewed.

Open Access This is an Open Access article distributed in accordance with the Creative Commons Attribution Non Commercial (CC BY-NC 4.0) license, which permits others to distribute, remix, adapt, build upon this work noncommercially, and license their derivative works on different terms, provided the original work is properly cited and the use is non-commercial. See: http:// creativecommons.org/licenses/by-nc/4.0/

\section{REFERENCES}

1. Lambert SD, Loiselle CG. Health information seeking behavior. Qual Health Res 2007;17:1006-19.

2. van der Molen B. Relating information needs to the cancer experience: 1. Information as a key coping strategy. Eur J Cancer Care (Engl) 1999;8:238-44.

3. Jefford $\mathrm{M}$, Tattersall $\mathrm{MH}$. Informing and involving cancer patients in their own care. Lancet Oncol 2002;3:629-37.

4. Fallowfield L. Participation of patients in decisions about treatment for cancer. BMJ 2001;323:1144.

5. Nagler RH, Gray SW, Romantan A, et al. Differences in information seeking among breast, prostate, and colorectal cancer patients: results from a population-based survey. Patient Educ Couns 2010;81 (Suppl):S54-62.

6. Nagler RH, Romantan A, Kelly BJ, et al. How do cancer patients navigate the public information environment? Understanding patterns and motivations for movement among information sources. $J$ Cancer Educ 2010;25:360-70.

7. Rutten LJ, Arora NK, Bakos AD, et al. Information needs and sources of information among cancer patients: a systematic review of research (1980-2003). Patient Educ Couns 2005;57:250-61.

8. Ziebland S, Chapple A, Dumelow C, et al. How the internet affects patients' experience of cancer: a qualitative study. BMJ 2004;328:564.

9. McCaughan E, Parahoo K, Prue G. Comparing cancer experiences among people with colorectal cancer: a qualitative study. J Adv Nurs 2011;67:2686-95.

10. Carlsson M. Cancer patients seeking information from sources outside the health care system. Support Care Cancer 2000;8:453-7.

11. Case DO, Andrews JE, Johnson JD, et al. Avoiding versus seeking: the relationship of information seeking to avoidance, blunting, coping, dissonance, and related concepts. J Med Libr Assoc 2005;93:353-62.

12. Ramanadhan $\mathrm{S}$, Viswanath $\mathrm{K}$. Health and the information nonseeker: a profile. Health Commun 2006;20:131-9. 
13. Czaja R, Manfredi C, Price J. The determinants and consequences of information seeking among cancer patients. J Health Commun 2003;8:529-62.

14. Miller SM. Monitoring and blunting: validation of a questionnaire to assess styles of information seeking under threat. J Pers Soc Psychol 1987;52:345-53.

15. Mills ME, Sullivan K. The importance of information giving for patients newly diagnosed with cancer: a review of the literature. J Clin Nurs 1999;8:631-42.

16. Leydon GM, Boulton M, Moynihan C, et al. Faith, hope, and charity: an in-depth interview study of cancer patients' information needs and information-seeking behavior. West J Med 2000;173:26-31.

17. Rutten LJ, Squiers L, Hesse B. Cancer-related information seeking: hints from the 2003 Health Information National Trends Survey (HINTS). J Health Commun 2006;11(Suppl 1):147-56.

18. Mistry $A$, Wilson $S$, Priestman $T$, et al. How do the information needs of cancer patients differ at different stages of the cancer journey? A cross-sectional survey. JRSM Short Rep 2010;1:30.

19. Smith-McLallen A, Fishbein M, Hornik RC. Psychosocial determinants of cancer-related information seeking among cancer patients. J Health Commun 2011;16:212-25.

20. Brown SP, Ganesan S, Challagalla G. Self-efficacy as a moderator of information-seeking effectiveness. J Appl Psychol 2001;86:1043-51.

21. Hashimoto $\mathrm{H}$, Fukuhara $\mathrm{S}$. The influence of locus of control on preferences for information and decision making. Patient Educ Couns 2004:55:236-40.

22. Tan AS, Nagler RH, Hornik RC, et al. Evolving information needs among colon, breast, and prostate cancer survivors: results from a longitudinal mixed-effects analysis. Cancer Epidemiol Biomarkers Prev 2015;24:1071-8.

23. Lambert SD, Loiselle CG, Macdonald ME. An in-depth exploration of information-seeking behavior among individuals with cancer: part 1: understanding differential patterns of active information seeking. Cancer Nurs 2009;32:11-23; quiz 24-5.

24. Lambert SD, Loiselle CG, Macdonald ME. An in-depth exploration of information-seeking behavior among individuals with cancer: part 2: understanding patterns of information disinterest and avoidance. Cancer Nurs 2009;32:26-36.

25. Lazarus RS, Folkman S. Stress, appraisal, and coping. New York: Springer, 1984

26. Johnson JD. Cancer-related information-seeking. Cresskill, NJ: Hampton, 1997.

27. Lenz ER. Information seeking: a component of client decisions and health behavior. ANS Adv Nurs Sci 1984;6:59-72.

28. Eheman CR, Berkowitz Z, Lee J, et al. Information-seeking styles among cancer patients before and after treatment by demographics and use of information sources. J Health Commun 2009;14:487-502.

29. Vogel BA, Bengel J, Helmes AW. Information and decision making: patients' needs and experiences in the course of breast cancer treatment. Patient Educ Couns 2008;71:79-85.

30. Germeni E, Schulz PJ. Information seeking and avoidance throughout the cancer patient journey: two sides of the same coin? A synthesis of qualitative studies. Psychooncology 2014;23:1373-81.

31. Miller SM. Monitoring versus blunting styles of coping with cancer influence the information patients want and need about their disease. Implications for cancer screening and management. Cancer 1995;76:167-77.

32. Garvin BJ, Moser DK, Riegel B, et al. Effects of gender and preference for information and control on anxiety early after myocardial infarction. Nurs Res 2003;52:386-92.

33. Coyne IT. Sampling in qualitative research. Purposeful and theoretical sampling; merging or clear boundaries? J Adv Nurs 1997;26:623-30.

34. Sandelowski M. Sample size in qualitative research. Res Nurs Health 1995;18:179-83.

35. Britten N. Qualitative interviews in medical research. BMJ 1995;311:251-3.

36. Strauss AL, Corbin JM. Basics of qualitative research: grounded theory procedures and techniques. Newbury Park, CA: Sage, 1990.

37. Sbaraini A, Carter SM, Evans RW, et al. How to do a grounded theory study: a worked example of a study of dental practices. BMC Med Res Methodol 2011;11:128.

38. Calman L, Brunton L, Molassiotis A. Developing longitudinal qualitative designs: lessons learned and recommendations for health services research. BMC Med Res Methodol 2013;13:14

39. Dickson-Swift V, James EL, Kippen S, et al. Doing sensitive research: what challenges do qualitative researchers face? Qual Res 2007;7:327-53

40. Murray SA, Kendall M, Carduff E, et al. Use of serial qualitative interviews to understand patients' evolving experiences and needs. BMJ 2009;339:b3702.

41. Wray N, Markovic M, Manderson L. "Researcher saturation": the impact of data triangulation and intensive-research practices on the researcher and qualitative research process. Qual Health Res 2007; 17:1392-402

42. Ortiz SM. Leaving the private world of wives of professional athletes: a male sociologist's reflections. J Cont Ethnogr 2004;33:466-87.

43. Germeni E, Sarris M. Experiences of cancer caregiving in socioeconomically deprived areas of Attica, Greece. Qual Health Res 2015;25:988-95. 